\title{
Perú: performance regional de la economía y la salud en el contexto del Coronavirus
}

\section{Peru: regional performance of the economy and health in the context of the Coronavirus}

\author{
Rudy Meyer Laguna Inocente ${ }^{1}$
}

\begin{abstract}
This paper analyzes the impacts of Covid 19 on the regional economy/health of Peru jointly, measured by the Covid 19 Regional Performance Index (IPCR19). The research shows that the degree of impact on the IPCR19 is explained by conjunctural and structural variables. The main variables that explain why the regions have been more or less affected by the pandemic are the acquisition of oxygen to contain the coronavirus, the availability of water in the home, rurality or lower population density, and the willingness to have buffer economic sectors with significant participation in GDP, mainly agriculture, hydrocarbons, mining, fishing and aquaculture activities.
\end{abstract}

Keywords: Covid 19, GDP, economy, health, regional, oxygen, water availability, rurality, population density, buffer economic sectors

\section{RESUMEN}

El presente estudio tiene como objetivo analizar los impactos del Covid 19 en la economía y en la salud regional peruana de manera conjunta, medido mediante el Índice Performance Regional Covid 19 (IPCR19). La investigación muestra que el grado de impacto sobre el IPCR19 está explicado por variables coyunturales y estructurales. Las principales variables que explican que las regiones hayan sido más o menos afectadas por la pandemia son la adquisición de oxígeno para contener el coronavirus, la disponibilidad de agua en el hogar, la ruralidad o menor densidad poblacional y la disposición de contar con sectores económicos amortiguadores con importante participación en el PBI, principalmente agropecuario, hidrocarburos, minería, pesca y acuicultura.

Palabras clave: Covid 19, PBI, economía, salud, regional, oxígeno, disponibilidad de agua, ruralidad, densidad poblacional, sectores económicos amortiguadores

Received: 20 de agosto del 2021

Accepted: 18 de setiembre de 2021

\section{Introducción}

El Perú y el mundo se encuentran enfrentando una de las etapas más complejas de la historia, el Covid 19. La economía y la salud pública internacional están siendo afectadas por la pandemia generada por el Coronavirus. De acuerdo al Fondo Monetario Internacional, en el año 2020 la economía mundial disminuyó -3.5\%, por la menor producción de las economías desarrolladas $(-4.9 \%)$, y de las economías emergentes y en desarrollo (-2.4\%). Estado Unidos, Europa, y América Latina y el Caribe cayeron -3.4\%, $-7.2 \%$ y $-7.4 \%$, respectivamente.

En relación a la salud afectada por el Covid 19, de acuerdo a la Universidad de Johns Hopkins, en el año 2020 se registraron a nivel mundial en promedio 23 personas fallecidas por cada 100 mil habitantes. Estados Unidos, Europa y Sudamérica registraron 106, 73 y 84 personas fallecidas por cada 100 mil habitantes, respectivamente.

En el ámbito local, en el año 2020 la economía peruana se contrajo en $-11.1 \%$ y, de acuerdo a la Universidad de Johns Hopkins, se registraron 114 personas fallecidas por cada 100 mil habitantes. En este mismo período, de acuerdo al Ministerio de Salud (MINSA) y al Sistema Informático Nacional de Defunciones (SINADEF), las personas fallecidas fueron 36,178 y 103,955, respectivamente; es decir, 111 personas en el primer caso y 319 personas en el segundo caso por cada 100 mil habitantes. La cantidad de personas fallecidas registradas por SINADEF corresponden al año 2020, menos el promedio de los tres años anteriores.

A nivel de regiones del país, en términos económicos, Madre de Dios fue la de mayor contracción del año 2020 (-23.6\%) y Moquegua es la única región del país que ha mostrado crecimiento $(1.7 \%)$. En relación a la salud, de acuerdo al SINADEF, Lima y Callao y Amazonas son las zonas del país con mayor y menor impacto, con 498 y 60 personas fallecidas por 100 mil habitantes, respectivamente.

El impacto regional diferenciado del Coronavirus es resultado de factores coyunturales y estructurales. En la presente investigación, mediante modelos de variables discretas, se evalúa la performance regional conjunta de la economía y la salud en el contexto del Covid 19. Para tal fin, se construye el Índice Performance Regional Covid 19 (IPCR19), el cual incorpora el crecimiento económico y la cantidad de personas fallecidas por 100 mil habitantes de acuerdo al SINADEF.

${ }^{1}$ Universidad de San Martín de Porres, Peru. E-mail:rlagunai@usmp.pe

How to cite: Laguna Inocente, R. M. (2022). Perú: performance regional de la economía y la salud en el contexto del Coronavirus. ANÁLISIS ECONÓMICO Y FINANCIERO, 5(1), 26-32. https://doi.org/10.24265/raef.2022.v5n1.49 
En este contexto, uno de los principales objetivos del estudio es determinar los factores que explican la performance regional sobre la economía y la salud, distinguiendo las regiones Menos y Más Afectadas. En las siguientes secciones del documento se desarrolla el método para alcanzar estos objetivos, inicialmente con una contextualización del problema, los instrumentos cuantitativos a ser utilizados, descripción de la data y los principales resultados. En la sección final del documento se presentan las principales conclusiones del estudio.

\section{Método}

\section{Contextualización}

El Covid 19 ha tenido efectos diferentes en cada región del país en el año 2020. En las siguientes figuras se muestran el crecimiento económico y la cantidad de personas fallecidas por 100 mil habitantes en cada una de las regiones del Perú. A partir de ambas variables se define el Índice Performance Regional Covid 19 (IPCR19), con valor de uno (1) para las regiones Menos Afectadas y cero (0) para las regiones Más Afectadas por el Coronavirus.

Como se puede observar, Madre de Dios es la región de mayor contracción económica del año 2020, disminuyó en $-23.6 \%$, más del doble del promedio nacional $(-11.1 \%)$. Moquegua ha sido la única región del país que ha mostrado crecimiento, $1.7 \%$. En relación a la salud, de acuerdo al SINADEF, Lima y Callao y Amazonas son las zonas del país con más y menos impacto, con 498 y 60 personas fallecidas por 100 mil habitantes, respectivamente.

\section{Figura 1 y 2}

Un primer aspecto a evaluar es la estructura económica regional que presenta el país. Las regiones de la zona costera se encuentran vinculadas principalmente a actividades de agricultura, comercio, construcción, manufactura, pesca, servicio, transporte y turismo. En esta zona del país prevalecen las zonas urbanas, se encuentran las ciudades más pobladas y con mayor interacción de personas del país. En la zona sierra altoandina del Perú prevalece actividades como agricultura, comercio, energía (hidroeléctricas), minería y turismo. En la amazonia peruana se concentran actividades agrícolas, comercio, energía (hidrocarburos), forestal, servicio y turismo.

Asimismo, en la contención del Coronavirus, la actuación de los tres niveles de gobierno (nacional, regional y municipal) ha tendido diferentes efectos sobre la performance regional durante el 2020. Una de las principales actividades para la contención de la pandemia y atenuar sus efectos sobre la población en las regiones ha sido a la adquisición de oxígeno, para ser utilizado en las Camas de Unidades de Cuidados Intensivos (Camas $\mathrm{UCI}$ ) o en los hogares ante la falta de disponibilidad de las Camas $\mathrm{UCl}$ en los establecimientos de salud de las regiones.

\section{Instrumentos}

Para explicar la performance regional en materia económica y la salud durante el primer año de pandemia, medido con el Índice Performance Regional Covid 19, utilizaremos modelos de variables discretas, especificamente modelos de variables binarias dependientes, también denominados modelos probabilísticos. Es decir, se explica el comportamiento de la variable dependiente $y_{i}=$ Índice Performance Regional Covid 19 con valores de uno (1) para las regiones Menos Afectadas y cero (0) para las regiones Más Afectadas por el Coronavirus. Las variables explicativas están representadas con $x_{i}^{\prime}=\left(c, x_{1 i}, x_{2 i}, x_{3 i}, x_{4 i}\right)$. El subíndice $i$ representa a cada una de las 24 regiones del Perú. Así, cuando es igual a uno (1) representa a una de las regiones Menos Afectadas y cuando es igual a cero (0) representa a una de las regiones Más Afectadas por el Coronavirus. Las variables explicativas se presentan a continuación:

- $x_{1 i}=$ Disponibilidad de agua en el hogar

- $x_{2 i}=$ Cantidad de óxigeno

- $x_{3 i}=$ Ruralidad

- $x_{4 i}=$ Amortiguador económico sectorial

La probabilidad de ocurrencia de $y_{i}$ se expresa como:

$$
\begin{aligned}
& \operatorname{prob}\left(y_{i}=1\right)=F\left(x_{i}^{\prime} \beta\right) \\
& \operatorname{prob}\left(y_{i}=0\right)=1-F\left(x_{i}^{\prime} \beta\right)
\end{aligned}
$$

La elección de la distribución $\mathrm{F}$ debe ser tal que se cumpla el criterio de probabilidad:

$$
\lim _{x^{\prime} \beta \rightarrow \infty} \operatorname{prob}\left(y_{i}=1\right)=1 \text { y } \lim _{x^{\prime} \beta \rightarrow-\infty} \operatorname{prob}\left(y_{i}=1\right)=0
$$

De acuerdo al tipo de distribución de probabilidad o de cuantía se distinguen comúnmente dos tipos de modelos. El primero de ellos es el modelo Probit, con una función de probabilidad acumulada F que sigue una distribución normal estándar. El segundo es el modelo Logit con una función de probabilidad acumulada $F$ que sigue una distribución logística. A continuación, se presentan las probabilidades para ambos modelos.

- Modelo probit

$$
\operatorname{prob}\left(y_{i}=1\right)=\Phi\left(x_{i}^{\prime} \beta\right)=\int_{-\infty}^{x_{i}^{\prime} \beta} \frac{1}{\sqrt{2 \pi}} \exp \left(-\frac{z^{2}}{2}\right) d z
$$

- Modelo logit

$$
\operatorname{prob}\left(y_{i}=1\right)=\Lambda\left(x_{i}^{\prime} \beta\right)=\frac{e^{x_{i}^{\prime} \beta}}{1+e^{x_{i}^{\prime} \beta}}
$$

Al ser modelos no lineales se utiliza el método de máxima verosimilitud para obtención de los parámetros :

$$
\begin{aligned}
L & =\operatorname{prob}\left(Y_{1}=y_{1}, Y_{2}=y_{2}, \cdots, Y_{n}=y_{n}\right) \\
& =\prod_{y_{i}=0}\left[1-F\left(x_{i}^{\prime} \beta\right)\right] \prod_{y_{i}=1} F\left(x_{i}^{\prime} \beta\right) \\
& =\prod_{i=1}^{n}\left[F\left(x_{i}^{\prime} \beta\right)\right]^{y_{i}}\left[1-F\left(x_{i}^{\prime} \beta\right)\right]^{1-y_{i}} \\
\ln L & =\sum_{i=1}^{n}\left[y_{i} \ln F\left(x_{i}^{\prime} \beta\right)+\left(1-y_{i}\right) \ln \left(1-F\left(x_{i}^{\prime} \beta\right)\right)\right]
\end{aligned}
$$


Al optimizar los parámetros $(\beta)$ se tiene la condición de primer orden:

$$
\frac{\partial \ln L}{\partial \beta}=\sum_{i=1}^{n}\left[\frac{y_{i} f_{i}}{F_{i}}+\left(1-y_{i}\right) \frac{-f_{i}}{\left(1-F_{i}\right)}\right] x_{i}=0
$$

\section{Descripción de la Data}

El Índice Performance Regional Covid $19 y_{i}$ es la variable binaria dependiente obtenida a partir de la combinación de valores normalizados del crecimiento económico y la cantidad de personas fallecidas por el Coronavirus por cada 100 mil habitantes. Los valores binarios han sido obtenidos a partir de la suma de los valores normalizados del crecimiento económico regional y la cantidad de personas fallecidas por cada 100 mil habitantes, en ambos casos mayor y menor a 0.50 , respectivamente. Así, cuando una región ha tenido en el año 2020 un crecimiento económico mayor al promedio regional y a su vez una menor cantidad de personas fallecidas por cada 100 mil habitantes (en relación al promedio regional), el valor del Índice Performance Regional Covid 19 es igual a uno (1); en cualquier otro caso, dicho valor es igual a cero (0).

La variable $x_{1 i}$ es la cantidad de oxígeno, medido por el volumen disponible para atención del Coronavirus en metros cúbicos por habitante. La variable explicativa $x_{2 i}$ es la disponibilidad de agua en el hogar, medido como el porcentaje de personas de cada región que tienen el servicio dentro de la vivienda. La variable $x_{3 i}$ es la medida de ruralidad de la región, y es binaria con valores de uno (1) cuando más del $50 \%$ de la población de la región habita en zonas rurales, caso contrario toma valores de cero (0).

La variable $x_{3 i}$ binaria es un proxi del grado de interacciones o contactos de las personas en cada una de las regiones; cuando mayor es la interacción o los contactos de las personas mayor es la probabilidad de contagio y, consecuentemente, de fallecidos por el Coronavirus. Como se muestra en las siguientes figuras, en promedio, las regiones de mayor ruralidad presentan menor densidad poblacional medida en metros cuadrados por persona. Cuando mayor es la densidad población existe mayor probabilidad de interacción o contacto entre personas. Las primeras personas contagiadas y la aceleración de los contagios se observó principales en las zonas urbanas del país, y progresivamente se fue expandiendo a las zonas o regiones con mayor presencia de ruralidad (ver Anexo 1). La variable $x_{4 i}$ es un amortiguador económico sectorial que tienen algunas regiones del país por disponer de actividades económicas que se desarrollan con menos interacciones o contactos entre las personas o trabajadores, o porque laboran con altos estándares de seguridad que ha permitido una rápida adecuación al nuevo contexto de convivencia con el Coronavirus. Esta variable normalizada representa la contribución en el crecimiento económico regional de los sectores que han tenido mejor performance frente a la pandemia, incluyen agropecuario, hidrocarburos, minería, pesca y acuicultura.

Figura 3

\section{Resultados}

Con respecto al análisis descriptivo de todas las regiones del Perú, en la siguiente tabla se presentan los principales resultados de las estimaciones del modelo de variable binaria dependiente Índice Performance Regional Covid 19, explicada por las variables cantidad de oxígeno, disponibilidad de agua en el hogar, ruralidad y el crecimiento económico de los amortiguadores sectoriales de las regiones del Perú. Con excepción del coeficiente constante, todos los valores de beta son positivos, lo que significa que las variables explicativas tienen efectos positivos sobre el Índice Performance Regional Covid 19.

Tabla 1

\section{Conclusiones}

La presente investigación es una primera aproximación para identificar y cuantificar a las principales variables que explican los impactos diferenciados del Covid 19 en la economía y en la salud regional peruana. Se evidencia que el Covid 19 ha tenido impactos diferenciados en las regiones del Perú en el 2020.

La investigación muestra que el grado de impacto sobre el Índice Performance Regional Covid 19 está explicado por variables de gestión para contener la pandemia y por variables estructurales vinculadas al sector salud y al grado de interacción de las personas. Las principales variables que explican que las regiones hayan sido más o menos afectadas por la pandemia son la adquisición de oxígeno para contener el coronavirus, la disponibilidad de agua en el hogar, la ruralidad o menor densidad poblacional y la disposición de sectores económicos amortiguadores regionales asociados a las actividades agropecuario, hidrocarburos, minería, pesca y acuicultura

Para instrumentalizar la cuantificación del impacto del Coronavirus tanto en la economía como en la salud regional se ha construido Índice Performance Regional Covid 19, con valores iguales a uno (1) cuando las regiones han sido Menos Afectadas y valores iguales a cero (0) cuando las regiones han sido Más Afectadas.

En este contexto, ha sido una variable fundamental la cantidad de oxígeno para atención de las personas contagiadas con coronavirus, representado por el volumen en metros cúbicos por habitante. Las regiones con mayor cantidad de oxigeno por habitante han sido las Menos Afectadas durante el 2020. Asimismo, la disponibilidad de agua en el hogar, medido como el porcentaje de personas de cada región que tiene el servicio dentro de la vivienda, ha tenido un impacto favorable sobre las regiones, ha permitido que las regiones sean Menos Afectadas por el Coronavirus.

De la misma manera, la variable ruralidad o menor densidad poblacional ha contribuido en impactar menos a las regiones del Perú. En efecto, cuando menor es la interacción o los contactos de las personas, menor es la probabilidad de contagio $y$, consecuentemente, de fallecidos por el Coronavirus. En contraste, en regiones del país que tienen grandes zonas urbanas, con altas concentraciones e interacciones de personas, más rápido y mayor ha sido los contagios.

Otra variable que ha sido fundamental para que las regiones sean Menos Afectadas es amortiguador económico sectorial que disponen, por tener en sus respectivas circunscripciones actividades económicas que se desarrollan con menos 
interacciones o contactos entre las personas o trabajadores, o porque son sectores cuyos trabajadores laboran con altos estándares de seguridad que ha permitido una rápida adecuación al nuevo contexto de convivencia con el Coronavirus. En este sentido, han sido Menos Afectadas las regiones con importante participación en el PBI regional de sectores económicos amortiguadores asociados a las actividades agropecuario, hidrocarburos, minería, pesca y acuicultura.

\section{Referencias}

Betcherman, G., Giannakopoulos, N., Laliotis, I., Pantelaiou, I., Testaverde, M., y Tzimas, G. (2020). The Short-Term Impacts of the COVID-19 Lockdown on the Greek Labor Market. Banco Mundial. Policy Research Working Paper 9356.

Chen, S., Igan D., Pierri N. and Presbitero A., International Monetary Fund. Tracking the Economic Impact of COVID19 and Mitigation Policies in Europe and the United States (2020), Special Series on COVID-19.

Green, W. (1999). Econometric Analysis, Fifth Edition, Models for Discrete Choice, 663 - 755.

International Monetary Fund. World Economic Outlook Update. January 2021.

Instituto Nacional de Estadıstica e Informatica (INEI). Producto Bruto Interno por Departamentos (Data Anual).

Instituto Nacional de Estadıstica e Informatica (INEI). Poblacion por Departamentos 2020.

Instituto Nacional de Estadıstica e Informatica (INEI). Porcentaje de poblacion Urbana y Rural por Departamentos.

Instituto Nacional de Estadıstica e Informatica (INEI). Porcentaje de personas por Departamentos que dispone de servicio de agua dentro de la vivienda.

Johnston y Dinardo (1997). Econometric Methods, Discrete and Limited Dependent Variable Models, $412\{452$.

Ministerio de Economıa y Finanzas (MEF). Transparencia Economica. Gasto de Inversion Publica de Gobierno Regionales (Data Anual).

Ministerio de Salud. Situacion Actual COVID19 Peru 20202021. 12 de Abril, pagina 31.

Ministerio de Salud. Documento Tecnico: Plan de preparacion y respuesta ante posible segunda ola pandemica por Covid19 en el Peru. RM N 928-2020/MINSA). Lima, Peru 2020.

Nicolas L., Bottan D., Vera-Cossio, B. (2020). The Unequal Impact of the Coronavirus Pandemic: Evidence from Seventeen Developing Countries. Paper No IDB-DP-785.

Tuan A. and Manh-Hung N. (2020). \COVID-19, lockdown and labor uncertainty" Working Paper 1137, 25-26.

Timothy H., Huy N. and Randa S., Determinants of PrePandemic Demand for the IMF's Concessional Financing, IMF Working Paper WP/21/15, 9-10.

Universidad de Johns Hopkins, Ourworldindata, Statistics and Research, Coronavirus (COVID-19) Cases (2020), https: //ourworldindata.org/covid-cases. 


\section{Lista de figuras}

Figura 1: Crecimiento económico y cantidad de personas fallecidas por regiones en Peú

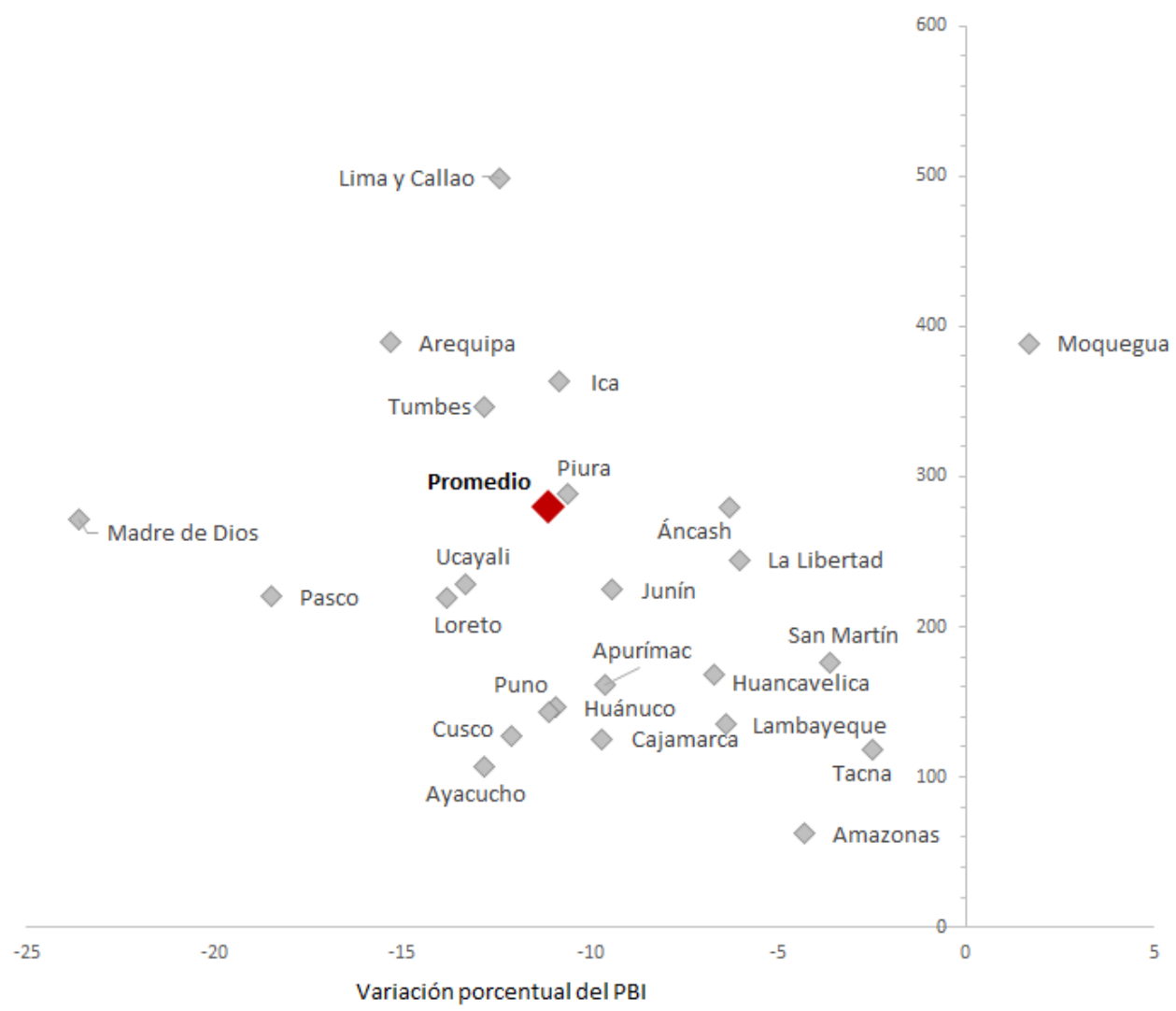

Figura 2: Índice Performance Regional Covid 19 (IPCR19)

\begin{tabular}{ll}
\multicolumn{2}{c}{ Menos Afectadas (11) } \\
Amazonas & \multicolumn{1}{c}{ Junín } \\
Áncash & La Libertad \\
Apurímac & Lambayeque \\
Cajamarca & San Martín \\
Huancavelica & Tacna \\
Huánuco & \\
\hline \multicolumn{1}{c}{ Más Afectadas (13) } \\
Arequipa \\
Ayacucho \\
Cusco \\
Ica \\
Lima y Callao \\
Loreto \\
Madre de Dios
\end{tabular}


Figura 3: Variables explicativas

\section{Cantidad de oxígeno para atención del Coronavirus \\ (Metros cúbicos por habitante)}

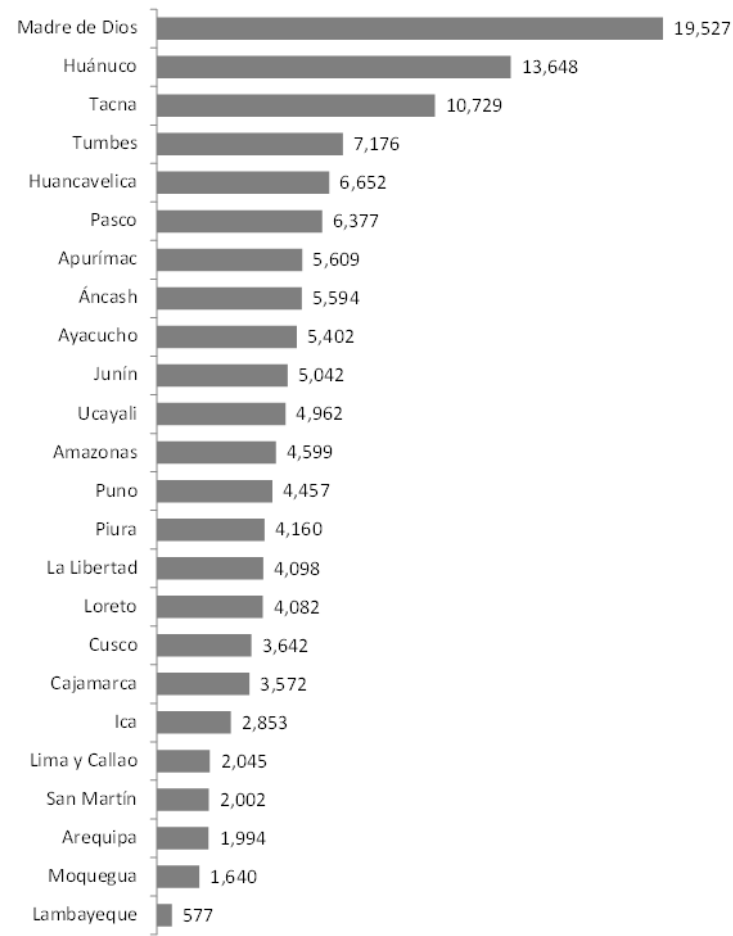

Ruralidad regional

(Porcentaje de la población)

\section{Disponibilidad de agua en el hogar}

(Porcentaje de la población)

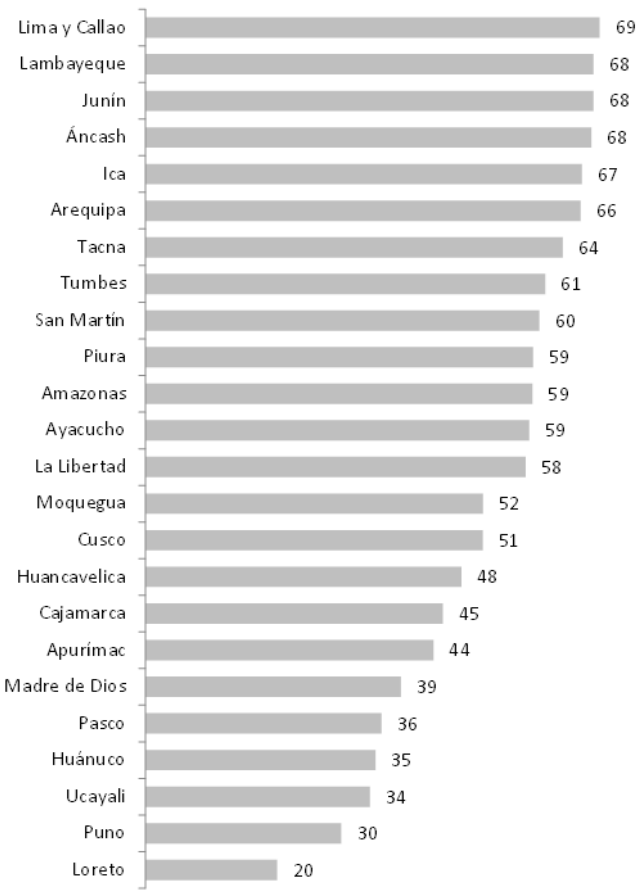

Amortiguador económico sectorial por región

(Variación porcentual real 2020)

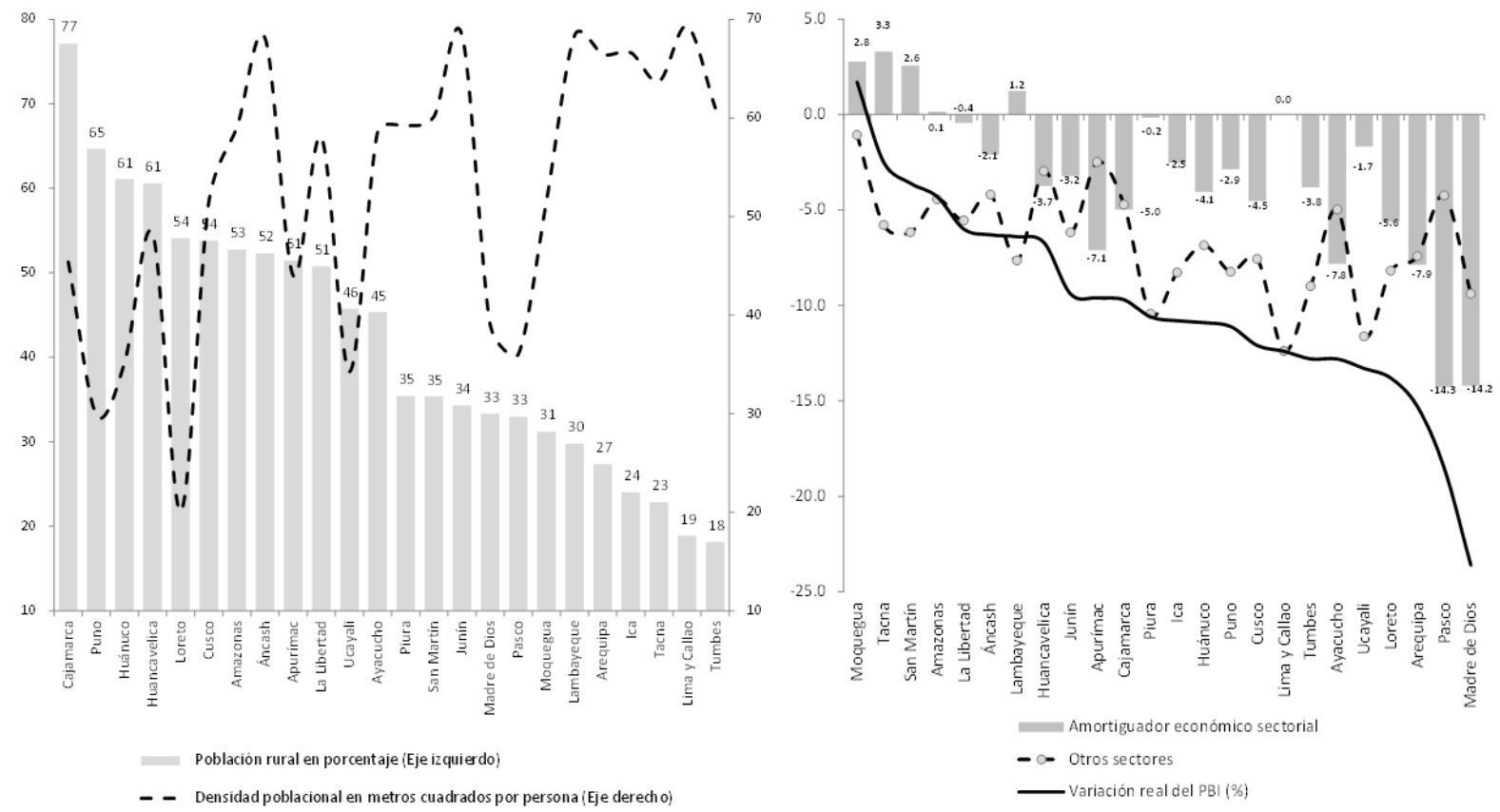




\section{Lista de tablas}

Tabla 1: Resultados

\begin{tabular}{|c|c|c|c|c|c|c|}
\hline \multirow{2}{*}{ Variables explicativas } & \multicolumn{3}{|c|}{ Modelo Probit } & \multicolumn{3}{|c|}{ Modelo Logit } \\
\hline & Beta & t-Statistic & P-Value & Beta & t-Statistic & P-Value \\
\hline Contante & -11.20958 & -2.098283 & 0.0359 & -19.60524 & -1.960546 & 0.0499 \\
\hline$x_{1 i}:$ Cantidad de oxígeno & 0.00024 & 1.320126 & 0.1868 & 0.00039 & 1.191668 & 0.2334 \\
\hline$x_{2 i}:$ Disponibilidad de agua en el hogar & 0.11695 & 1.731996 & 0.0833 & 0.18817 & 1.695034 & 0.0901 \\
\hline$x_{3 i}:$ Ruralidad & 3.45753 & 2.228196 & 0.0259 & 6.19527 & 2.002500 & 0.0452 \\
\hline$x_{4 i}:$ Amortiguador económico sectorial & 4.18879 & 1.495744 & 0.1347 & 7.35706 & 1.421974 & 0.1550 \\
\hline McFadden R-squared & 0.508182 & & & 0.504737 & & \\
\hline Log likelihood & -8.140629 & & & -8.197641 & & \\
\hline
\end{tabular}

